\title{
Large-Scale Implementation of Collaborative Care Management for Depression and Diabetes and/or Cardiovascular Disease
}

\author{
Arne Beck, PhD, Jennifer M. Boggs, MSW, Angelika Alem, MPH, \\ Karen J. Coleman, PhD, Rebecca C. Rossom, MD, Claire Neely, MD, \\ Mark D. Williams, MD, Robert Ferguson, and Leif I. Solberg, MD
}

Background: Collaborative care models have been shown to improve mental and physical health, but their effectiveness varies. Implementation science frameworks identify measures at the structural (eg, sociocultural context, public policies), organizational, provider, innovation, and patient levels that may facilitate or impede collaborative care effectiveness.

Objective: To describe commonalities and variation in multilevel measures associated with the implementation of Care of Mental, Physical, and Substance-Use Syndromes (COMPASS), a large-scale collaborative care intervention for depression, diabetes, and cardiovascular disease.

Design: Qualitative study using semistructured descriptive data obtained from annual site visit reports and supplemental site surveys.

Participants: COMPASS care teams from 8 health care systems serving 3854 patients with active depression and poorly controlled diabetes and/or cardiovascular disease.

Intervention: COMPASS included weekly case reviews with a consulting physician and psychiatrist, a patient-tracking registry, and monitoring of hospital and emergency department use.

Main Measures: Site visit reports were analyzed with Atlas.ti software to qualitatively describe implementation measures and their variation across sites.

Key Results: Nine measures were identified that impacted implementation efforts across health systems: (1) challenges in health systems' organizational environments, (2) prior care coordination experience, (3) physician engagement, (4) care team trust and cohesion, (5) care manager training and experience, (6) patient enrollment length, attainment of clinical targets, and frequency/content of care manager contacts, (7) patient-tracking registries, (8) quality improvement and outcomes monitoring reports, and (9) patients' social needs.

Conclusions: Understanding multilevel measures impacting COMPASS implementation could increase the success of future collaborative care implementation efforts. (J Am Board Fam Med 2018;31:

702-711.)

Keywords: Cardiovascular Diseases, Depression, Diabetes Mellitus, Organizational Innovation, Quality Improvement, Registries, Surveys and Questionnaires

Collaborative care management (CCM) involving behavioral health and primary care providers (PCPs) entails a structured, multicomponent approach using principles from the chronic care

This article was externally peer reviewed.

Submitted 2 March 2017; revised 30 April 2018; accepted 14 May 2018.

From Kaiser Permanente Colorado Institute for Health Research, Denver, CO (AB, JMB); Department of Research and Evaluation, Kaiser Permanente Southern California, Pasadena, CA (AA, KJC); Institute for Clinical Systems model, ${ }^{1}$ including consultation and decision support to PCPs from mental health specialists. Typical collaborative care components include the use

Improvement, Bloomington, $\mathrm{MN}(\mathrm{CN})$; Psychiatry and Psychology Division of Integrated Behavioral Health, Mayo Clinic, Rochester (MDW); Pittsburgh Regional Health Initiative, Pittsburgh, PA (RF); HealthPartners Institute, Minneapolis (RCR, LIS).

Funding: This project was supported by Grant 1C1CMS33-1048-01-00 from the Department of Health and Human Services, Centers for Medicare \& 
of evidence-based protocols for treatment initiation and adjustment, and strategies for engaging patients in treatment goal-setting, medication adherence, and self-management. These CCM components have demonstrated effectiveness in reducing depressive symptoms, ${ }^{2-7}$ levels of glycohemoglobin, low-density lipoprotein cholesterol, and systolic blood pressure (SBP), as well as improving care processes, for patients with depression and with co-occurring chronic medical conditions, such as diabetes, cardiovascular disease, and asthma. ${ }^{8-15}$

However, there are large variations in the effect size on outcomes of CCM models. ${ }^{2-4,13}$ These variations are likely due to differences in the implementation of CCM, including mode of contact (in person, phone, e-mail), intensity and/or frequency of contact, case load, staff characteristics, delivery models (a focus on medication vs behavior change using motivational interviewing and/or behavioral activation strategies), individual provider versus team approaches, and the types of health systems (integrated systems vs individual medical practices; fee-for-service vs capitated payment). $\mathrm{Pa}-$ tient characteristics such as disease severity and social nonmedical needs also contribute to variation in collaborative care effectiveness.

Implementation science provides several frameworks with which to organize the numerous factors accounting for variations in CCM effectiveness. ${ }^{16-18}$ A systematic review by Chaudoir et $\mathrm{al}^{19}$ of factors affecting implementation of health innovations broadly categorizes this variation within structural (public policies, political, social or economic climate), organizational (innovation climate, leadership effectiveness), provider (training, attitudes toward evidence-based practice), innovation (relative advantage, complexity), and patient (mo-

Medicaid Services. The contents of this publication are solely the responsibility of the authors and do not necessarily represent the official views of the US Department of Health and Human Services or any of its agencies. The research presented here was conducted by the awardee. Findings might or might not be consistent with or confirmed by the independent evaluation contractor.

Conflict of interest: All authors have no conflicts of interest to disclose, except Dr. Mark Williams, who received honoraria in the past 3 years from Acupera (a company creating software to support care coordination) and Neuroscience Education Institute (a company that creates CME materials).

Corresponding author: Arne Beck, PhD, Kaiser Permanente Colorado, Institute for Health Research, 2550 S. Parker Rd., suite 200, Aurora, CO 80014 (E-mail: Arne.beck@kp.org). tivation, severity of illness) levels, each of which can facilitate or impede implementation efforts.

Although variations in CCM models have been extensively described, ${ }^{20-29}$ research on their actual large-scale implementation is more limited. ${ }^{30-33}$ This article provides a qualitative description of implementation measures impacting a large-scale collaborative care model for patients with depression and comorbid diabetes and/or cardiovascular disease. The CCM model, Care of Mental, Physical, and Substance-Use Syndromes (COMPASS), was implemented within 8 health care systems across the United States over a 3 -year period.

\section{Methods}

\section{Setting and Participants}

The COMPASS model has been described elsewhere. ${ }^{15,34,35}$ Briefly, COMPASS was supported by a 3-year award (2012 to 2015) from the Center for Medicare and Medicaid Innovation to implement and evaluate health care innovations. COMPASS was led by the Institute for Clinical Systems Improvement and implemented through a collaboration of 10 COMPASS partners, including 8 implementation partners and 2 supporting partners, representing 18 medical groups and over 172 clinics across 8 states. A total of 3854 patients were recruited into COMPASS between February 2013 and March 2015. Patients were eligible for COMPASS if they had active depression (PHQ9 of at least 10) ${ }^{36}$ and one of the following poorly controlled medical conditions: diabetes mellitus with an HgbA1c $>8 \%$ or SBP $>145$, and/or cardiovascular disease with SBP $>145$. Patients over 65 years of age were also eligible for COMPASS if they had active depression and SBP $>160$.

Each implementing site received institutional review board approval for the research and evaluation portions of the COMPASS implementation.

\section{COMPASS Intervention Components}

COMPASS partners were asked to adhere to the following core components in their implementation efforts: (1) a clearly defined care management process with a care team of at least one care manager (CM), physician consultant, and psychiatrist who conducted weekly systematic case reviews (SCR), focusing on treat-to-target guidelines; (2) a care management tracking system (CMTS) to track 
patients for follow-up contacts and to collect information for routine aggregate and site-specific quality improvement (QI) reports; and (3) monitoring of hospital and emergency department use to prevent unnecessary readmissions.

\section{Data Collection}

Semistructured interview and observational note data on implementation across the 18 COMPASS medical groups were obtained from reports written by a team of Institute for Clinical Systems Improvement practice coaches between 6 months and 1 year following site visits. A key aim of the site visits was to understand local variation in the adoption and implementation of core model components and methods used by sites for improving outcomes of COMPASS. The site visit team interviews included the following topics: motivation to implement COMPASS, COMPASS's alignment with the organization's mission/values, integration with or complementarity to other care management initiatives, adaptations in other parts of health system due to COMPASS, barriers and successes encountered, successful approaches to patient selfmanagement, response from the health system to the SCR, barriers/successes with the SCR, how the care team used and responded to QI data, and the sustainability of COMPASS. In addition to the care team interview, each medical group's SCR was observed and follow-up questions about the process were asked of the full interdisciplinary team, including administrative leaders. Follow up one-onone interviews were conducted to gain deeper insight and included the following categories of inquiry: team composition and processes; patient case finding; systematic case review and recommendations to the PCP team; registry and use of data for action; care transitions, including ED/hospital usage; and leadership.

The site visit and SCR interviews were developed based on coaching call experiences and with input from the COMPASS executive committee.

Additional data were collected from each medical group following qualitative analyses of initial site visit reports to provide further clarification and to expand on issues identified. The survey contained the following questions: (1) Have PCPs at your site had prior experience with care management programs similar to COMPASS? (2) How did prior experience with care management (or lack thereof) help/hinder implementation of
COMPASS at your site? (3) Did you notice any change over time in how PCPs viewed COMPASS care at your site? (4) What has been the typical length of time that patients have been in COMPASS at your site? (5) What do you think is an appropriate length of time for patients to be in COMPASS care? (6) How did you transition patients back to usual care provided by primary care teams or other providers at your site, once you discharged them from COMPASS?

The site visit reports and follow-up surveys were designed to identify specific successes and challenges related to COMPASS implementation that were common or unique to the medical groups and that could provide guidance for further QI efforts. Although a formal implementation science framework was not used prospectively in the collection of the qualitative data, we found it informative to use multilevel framework from Chaudoir et $\mathrm{al}^{19}$ to categorize implementation measures that were identified.

\section{Analyses}

Considering the semistructured approach to the site visit and SCR interviews on a priori topics of interest, an inductive content analysis approach using memos was chosen. This approach is recommended when there are a priori theories, as opposed to a grounded theory approach where theory is expected to emerge from the data. ${ }^{37}$ Following the content analysis approach defined by Glaser \& Laudel, ${ }^{37}$ we started with creating broad categories of data that were relevant to our research questions about implementation variation across sites. Author $\mathrm{JMB}$, using Atlas.ti software, ${ }^{38}$ read and created 5 broad memos of site visit report content with supporting quotations (between site differences, COMPASS learning and impact to the health system, challenges to implementing COMPASS, background of $\mathrm{CMs}$, and contributors to the site visit reports). These were reviewed with author $A B$, and more specific qualitative measures were identified that are reported below in the results. A second review of the data was conducted by JMB using the specific measures (eg, length of patient enrollment in COMPASS, registry use) and new memos with supportive quotations were created for each of these final measures. Starting broad and then narrowing down to more specific measures is a suggested approach to ensure that the intended 
meaning of the information is not lost in the analysis. $^{37}$

\section{Results}

Measures from the site visit reports and surveys are described below, organized from broader to narrower levels of implementation, that is, structural, organizational, provider, innovation, and patient, that are described in the implementation framework by Chaudoir et al. ${ }^{19}$

\section{Structural and Organizational Level}

Existing or New Challenges in the Organizational Environment of the Health System

The COMPASS model aligned with organizational goals to reduce unnecessary hospital and emergency department use and cost, to measure patientreported outcomes related to pay-for-performance metrics, and among some health plans, to achieve quality of care ratings from national quality organizations (for example, National Committee for Quality Assurance) to which they were accountable. However, COMPASS implementation occurred during the initiation of the Accountable Care Act (ACA), resulting in a significant influx of new patients and problems with their access to care for many of the COMPASS sites. Some medical groups were also challenged with provider turnover within health systems as well as COMPASS leadership, and low morale among PCPs. Therefore, medical groups underestimated the length of time required to implement COMPASS, given the prevailing organizational and environmental pressures. As one medical group member mentioned: "To create and implement this model (COMPASS), 12 to 18 months is needed." Another challenge to COMPASS was adequate compensation for program services, as medical groups characterized by a fee-forservice model had less flexibility in care delivery approaches because of the requirement that services (such as physician time for the SCR or phonebased CM services) be billable under current coding rules. One medical group member mentioned, "(There is) no incentive to do phone medicine." Another mentioned that "There would need to be funding for Care Management. The biggest issue for us was the miscalculation of funding for care management." The weekly SCRs with the CMs and consulting physicians were also not reimbursable yet believed to be valuable: "The team sees a great value in getting the consulting psychiatrist's perspective and expertise. They hope to maintain this, but resources are an issue." Finally, all medical groups mentioned that although effectiveness of the program to reach treatment targets was an important and expected outcome of COMPASS, "proven cost savings" would likely be required to maintain COMPASS after grant funding ended.

\section{Prior or Concurrent Experiences with Care Coordination}

A site lead that implemented COMPASS across multiple medical offices commented: "COMPASS was the first care coordination model in many medical offices." Medical groups' prior or concurrent experiences with care management was an indicator of their readiness for implementation of COMPASS. Some groups with existing CM programs initially viewed COMPASS as either a competing program or one requiring changes to existing CM programs, requiring more effort than implementing COMPASS de novo. For medical groups with concurrent CM programs, it was important to invest in coordination and partnership with these programs as well as highlight the ways COMPASS was unique and not repetitive, as mentioned at one medical group: "COMPASS is unique because it is deeper, more emotional, more holistic, and more patient centered." A key distinction of COMPASS versus other CM programs was the patient-centered approach to goal setting (ie, lose weight, increase exercise, adhere to medications) and the use of a motivational interviewing approach to set collaborative goals with the patient.

\section{Engagement with PCPS}

A total of 1554 primary care clinicians from medical groups ranging in size from small (20 to 100 PCPs), to medium (101 to $200 \mathrm{PCPs})$, to large (201 to 1,000 PCPs) were eligible to enroll patients into COMPASS. Although the numbers of PCPs who referred patients to COMPASS were not formally tracked across sites, anecdotes from site visit reports suggested that referrals from PCPs varied substantially. A key measure from site visit reports and surveys was the level of engagement with local primary care teams, specifically on enrollment success, receipt of treatment recommendations from the SCR team, and continuation of COMPASS after grant funding ended. The level of PCP support was based on such factors as prior experience 
with CCM models, the CM having valuable information about patients to share, PCP familiarity (and therefore trust) with the CMs, and positioning COMPASS as a PCP-led model versus a CM-led model (eg, PCPs had final approval of recommended changes to the treatment plan made by the clinic physicians consulting on the COMPASS program). One team described the process for gaining PCP input on recommendations from the CMs this way: "Different PCP's vary in referrals and acceptance of recommendations. Recommendations are generally a 1-way process without response from the PCP."

Medical groups describing low engagement reported the reluctance of PCPs to cede some control of patient care to the COMPASS CM (placing more value on traditional medicine). There was also a perceived lack of benefit of CCM and proactive outreach based on identifying at-risk patients, or a perception about patient confidentiality concerns in a team-based model (ie, patient preference that only their doctor to know/talk about them). As one team commented: "This system is traditional in the respect of waiting for patients to access the health care system when needed, versus finding patients based on their medical conditions."

More highly engaged medical groups were more likely to have an established culture of team-based care with shared medical records between departments. In these systems, CM input and suggestions and SCR recommendations were supported by PCPs with little resistance. Although having COMPASS physician champions was important in some medical groups, others had few physician champions, but did have a culture that supported team-based models and shared responsibility for patient care.

Some medical groups started with a high level of engagement, others started low and gained trust over time, and a few struggled with low engagement throughout. Several strategies were suggested to build engagement with primary care teams. These included having clear documentation of the COMPASS CM notes, including patient goals and a self-management plan that the PCP could easily see in the medical record, and ensuring that the $\mathrm{CM}$ was viewed as adding value and taking on work that would otherwise be done by the PCP or the PCP team versus adding to the PCP's workload. Many sites discussed having to gain trust slowly by showing the primary care teams over time that
COMPASS was an added benefit that helped PCPs monitor and implement the treatment plan between physician visits, improving care and saving time in the long run.

\section{Team Dynamics}

The SCR team was a new type of team for most medical groups, with an increased level of interaction between medical and behavioral health professionals. Teams that dedicated time to improving their own processes demonstrated improved team accountability for progress. Important team dynamics included cohesiveness, trust, and team members feeling like valued contributors. An increase in the sense of "teamness" did occur for some CM teams over time. (Claire Neely, personal communication) Teams also reported challenges with accountability for maintaining fidelity to a scheduled weekly SCR with the associated costs and time commitments, as well as questions regarding which specific aspects of the COMPASS model were critical for outcomes and which could be adapted as local needs emerged.

\section{Provider Level}

\section{CM characteristics}

The background and training of COMPASS CMs varied across sites and included nurses, social workers, psychologists, licensed professional counselors, and pharmacists. Regardless of licensure, several $\mathrm{CM}$ attributes were seen as essential to success in the COMPASS model, including the ability to establish rapport quickly with patients, ability to exercise independent judgment in clinical problem solving, awareness of local services, and prior experience with the population (eg, Medicaid, diabetes). Depending on background and training, some CMs focused more on depression care by using behavioral intervention strategies such as motivational interviewing and behavioral activation, whereas others focused more on treating medical conditions to target as a primary goal. One CM commented that she "feels that behavioral approaches are what I do all the time. My focus has always been more on behavior than on medications."

\section{Innovation Level}

Patient Enrollment Length, Attainment of Clinical

Targets, and Frequency/Content of Care CM Contacts

The COMPASS model was designed to be temporary, in that once goals had been reached and re- 
lapse prevention plans were in place, the patient would be transitioned back to primary care. The model was not time limited, nor did it specify stringent requirements for patient transitions from enrolled to discharge to allow for local customization. The model recommended patients remain in the program until all targets were reached (PHQ9 $<5$, SBP $<140$ and diastolic blood pressure $<90$, $\mathrm{HbA1C}<8.0$ ), and a maintenance/relapse prevention plan had been collaboratively developed with the patient. Average length of patient enrollment at each medical group varied from 1 to 26 months. Some medical groups set a time limit (typically 6 to 12 months) to maintain tight control on patient caseloads (one CM noting that COMPASS enabled a "focus on what can to be done to effectively transfer back to PCP teams, rather than maintain long-term in care management."), but most kept patients enrolled for the program's duration. A key factor in enrollment length was the medical complexity of patients. Some patients may have reached all of the COMPASS targets but still desired continued care management to ensure gains were maintained. Patients who were discharged as soon as they reached their targets were sometimes reenrolled because they failed to maintain progress on their goals. This led most medical groups to lengthen enrollment and to develop different patient status categories that defined the intensity of contact: active, maintenance, lost to follow-up, and discharged.

Many SCR teams commented that although some patients did not attain all their COMPASS clinical targets, a number of patients felt successful in achieving improvements on those measures as well as achieving their own personal goals that were separate from but complementary to the COMPASS-defined clinical targets. SCR teams also felt that managing patients with advanced illness or multiple comorbidities (in addition to diabetes, depression, and cardiovascular disease) required longer term care management than the intended design of the COMPASS model. As one CM stated: "Patients have more comorbidity than just COMPASS conditions which lead to a more advanced disease state where it is hard to effect change." These beliefs about the need for longer patient enrollment in COMPASS were born out in objective data (published elsewhere) showing that depression remission and response rates were generally lower in patients who were enrolled 3 months or fewer compared with patients enrolled for longer periods. ${ }^{15}$

A related issue was the frequency of CM contact with patients and the variation in the content of these contacts. Some medical groups only called patients to check in on their response to and adherence with medications. Others focused strongly on self-care improvement goals in calls (such as using motivational interviewing to elicit the patient's own reasons for behavior changes related to diet, exercise, and other healthy activities). Regardless of CM content, those CMs who spent more time on COMPASS-related tasks had higher rates of depression improvement among their patients (results published elsewhere). ${ }^{35}$ Variation also occurred in the approach to difficult-to-reach patients: some SCR teams decided not to try to reach such patients or to discuss them in SCRs, whereas others took an aggressive approach to outreaching these patients.

\section{Registry Use}

The use of a CMTS to track and monitor patients' progress yielded mixed results. The majority of medical groups used a CMTS developed by the Advancing Integrated Mental Health Solutions (AIMS) Center registry. Six medical groups developed their own CMTS, most of which were embedded in their EMRs. In either case, use of the CMTS by CMs for clinical management was generally low across medical groups, and was viewed more as a technology for data collection for the project than a clinical management tool. The CMs using the AIMS Center CMTS were required to document patient data in both the CMTS and in their EMRs, generating some resistance to this extra effort. Perhaps due to these experiences, and to how health systems decide on which IT aspects to sustain, few medical groups indicated an intention to continue using the COMPASS CMTS when the project was completed.

Despite the impediments to CMTS usage, there was general acknowledgment of the need for significant data infrastructure to quantify the degree to which program processes and outcomes were accomplished, and to tie these metrics to payment to provide adequate incentive to ensure that program costs were warranted. In addition, this measure identified the need for more intensive training and coaching of COMPASS teams to engage them in registry-based population management. 
Use of QI Reports

Significant variation was observed in the use of the centrally produced QI reports, based on monthly submission of data from the medical groups. Most used the reports as is, a few did not use them at all, and $a$ few made more abbreviated shadow reports. A few medical groups reported regularly using the QI reports to drive changes in the delivery of their program, though the lack of trend data limited their usefulness. The reports themselves went through several revisions to improve accuracy and clarity, although some CM teams had difficulty in interpreting the data and questioned its accuracy. However, the concerns identified with the reports did spark QI responses to address reasons for inaccurate data.

One of the more challenging decisions made by the COMPASS collaborative was to agree to complete transparency of data across sites. Numerous concerns were raised by the 18 medical groups, including 1) the need to keep what could be competitive information confidential, 2) fear that lower performers would be devalued by the group, and 3) concern that data would be used primarily for performance evaluation rather than for QI. To allay these concerns, an explicit agreement was written to address these issues. By sharing identified results among health care partners and their medical groups, specific processes associated with better outcomes could be identified, studied, and shared throughout the collaborative. This "early signal" property of the collaborative enabled identification of key challenges, such as the higher than expected social needs of this population, facilitating a consortium-wide discussion, and resulting in additional educational support for the CMs, and shared strategies.

\section{Patient Level}

Patients' High Degree of Social Needs

In addition to medical and behavioral health needs, SCR teams noted a high degree of social needs among their patients, requiring additional time for helping arrange services related to transportation, food, and housing. Patients' limited resources also led to an adoption of different means of contact at some medical groups, such as the use of phone appointments. Many CMs felt that motivational interviewing was a good tool to engage patients in goal setting that honored their needs and prefer- ences. Site survey responses suggested that the use of one central CM to coordinate services for patients with complex needs resulted in more consistent feedback on treatment progress and increased patient satisfaction with their care.

The finding of a high degree of social needs among patients also prompted discussion of the need to modify the COMPASS model. Some medical groups used social workers as CMs and/or as an addition to the SCR team to facilitate patients' access to community resources. As one put it: "(it is) nice to have a cross-pollinated team of people: somebody with a social work background can be effective and learn the medical components pretty quickly."

\section{Discussion}

We used qualitative data to describe measures common and unique to health care systems that facilitated or impeded the large-scale implementation of COMPASS, a collaborative care model for patients with depression and comorbid diabetes and/or cardiovascular disease. These measures were categorized within a multilevel implementation framework ${ }^{19}$ to provide contextualization to other literature on the implementation of health innovations. ${ }^{16-18}$

At the structural and organizational levels, the influx of new patients due to the start of the Accountable Care Act was an external pressure that slowed COMPASS implementation at all sites. Regulations and legislation have been identified as impediments to implementation in literature reviews of determinants of innovation within health care organizations. ${ }^{39}$ In addition, broader dissemination of COMPASS was a challenge faced by fee-for-service health systems because COMPASS $\mathrm{CM}$ services were not reimbursable. Developing reimbursement mechanisms (eg, procedure codes) to bill for multidisciplinary CM services to serve patients with depression and medical comorbidities is an important issue that warrants consideration by the Centers for Medicare and Medicaid services. Other site-specific organizational measures were sociocultural in nature, including turnover within medical groups, provider morale, provider engagement, and team cohesion. Problems in these areas slowed COMPASS adoption and implementation, hampered collaboration between providers and $\mathrm{CM}$ teams, and negatively impacted CM perfor- 
mance. Staff turnover and capacity are organizational factors that also have been identified as implementation impediments. ${ }^{39}$ In addition, sites without CM experience were less prepared to implement COMPASS, whereas sites with CM experience had to adapt COMPASS to their existing CCM structure.

At the provider level, CM characteristics varied across sites by type of clinical training and licensure. Management of patients with depression and comorbid medical conditions required skills in both the behavioral health treatment (eg, motivational interviewing, behavioral activation) and medical management (eg, recommending treatment intensification to the PCP). Ceding of care to CMs by PCPs depended on providers' trust in CMs and their general level of engagement with COMPASS. Where trust was established and engagement was high, PCPs valued behavioral health, social work, and medical management support from CMs.

The COMPASS innovation itself impacted the implementation process and varied across sites. For example, the length of patient enrollment and frequency of $\mathrm{CM}$ contacts were influenced by patient complexity. One lesson from implementing COMPASS was that the intended length and frequency of care management may have been underestimated given the complexity of the patient population. Site variation was also observed in the use of patient tracking registries and QI reports. Although these technology tools were regarded as necessary for population management and monitoring care processes and outcomes, more emphasis on the end-user experience would have helped to increase their ease of use and interpretability of data reports.

Finally, the high degree of patients' social needs was a commonly identified measure across sites, requiring that CMs allocate additional time to arrange various social services for patients. Patients' social needs and their complexity related to the combination of mental health and medical comorbidities also increased CM burnout. ${ }^{40}$ Site variation in addressing patients' social needs included the use of social workers to facilitate access to community resources, use of motivational interviewing strategies to foster patient engagement, and use of phone appointments and centralized CM services to reduce patient costs.

In summary, our descriptive analysis identified commonalities as well as substantial variation in the implementation of COMPASS components across medical groups. Although variation in the implementation of health care innovations may be viewed as undesirable, it often reflects adaptation and tailoring of intervention components to fit resources and limitations of local practices. Adaptation of innovations is increasingly viewed as a necessary condition for the success of implementation efforts. ${ }^{16,41}$ Our findings were also consistent with those from similar studies of collaborative care implementation that highlight the importance of teamwork and collaboration, CM clinical skills, physician engagement, and patient data tracking for quality monitoring. . $^{34,42,43}$

Results from this qualitative description of COMPASS implementation may be useful to determine important areas of focus for future CCM implementation planning efforts, including:

1) Adjusting implementation timelines to realistically account for unanticipated factors external and internal to health care systems, for example, changes in health care policy, provider and leadership turnover, and practice disruption resulting from CCM implementation;

2) Fostering provider engagement in the CCM model (eg, communicating its benefit in improving outcomes for their complex patients) and investing in strategies to enhance provider morale to build $\mathrm{CM}$ team trust and cohesion. The use of practice coaches may be one approach to facilitate engagement and team cohesion;

3) Ensuring CM teams are comprised of members with adequate training and experience in behavioral intervention techniques, clinical management skills (eg, treat to target approaches), and knowledge of community resources to address patients' social needs;

4) Establishing specific criteria for the length of patient enrollment in care management and providing an adequate frequency of CM contacts for more complex patients;

5) Collaborating early with end users to develop user-friendly patient-tracking registries and interpretable QI and outcomes management reports.

We would like to express our gratitude to Dr. Wayne Katon, whose seminal work on collaborative care for depression and diabetes provided the guidance for our current work. 
We wish to acknowledge the following individuals as significant contributors to the manuscript preparation, for leadership from their respective organizations in the COMPASS project, and for contributing patients and collecting data for the project: Kris Ohnsorg, RN, MPH, and Emily from HealthPartners Institute, Minneapolis, MN; Tani Hemmila, MHA, BSW, from the Institute for Clinical Systems Improvement, Bloomington, MN; Robin Whitebird, PhD, MSW, from University of St Thomas, School of Social Work; Katherine Larson, RN, from Mayo Clinic, Psychiatry and Psychology Division of Integrated Behavioral Health, Rochester, MN; Mark Dreskin, MD, from Kaiser Permanente Southern California, Department of Research and Evaluation; Betsy Pollock, MSW, ACSW, LICSW, from Mount Auburn Cambridge Independent Practice Association; and Emily Brandenfels, MD, MS, from Community Health Plan of Washington.

This research was presented at the Health Care Systems Research Network Annual Conference in Atlanta, GA, April 15, 2016.

To see this article online, please go to: bttp://jabfm.org/content/ 31/5/702.full.

\section{References}

1. Bodenheimer T, Wagner EH, Grumbach K. Improving primary care for patients with chronic illness. JAMA 2002;288:1775-9.

2. Kwan B, Nease DE, Jr. The state of the evidence for integrated behavioral health in primary care. In: Talen MR, Burke Valeras A, eds. Integrated behavioral health in primary care. New York, NY: Springer; 2013:65-98. 3.

3. Gilbody S, Bower P, Fletcher J, Richards D, Sutton AJ. Collaborative care for depression: a cumulative meta-analysis and review of longer-term outcomes. Arch Intern Med 2006;166:2314-21.

4. Thota AB, Sipe TA, Byard GJ, et al. Collaborative care to improve the management of depressive disorders: A community guide systematic review and meta-analysis. Am J Prev Med 2012;42:52538.

5. Katon W, Von Korff M, Lin E, et al. Stepped collaborative care for primary care patients with persistent symptoms of depression: a randomized trial. Arch Gen Psychiatry 1999;56:1109-15.

6. Unutzer J, Katon W, Callahan CM, et al. Collaborative care management of late-life depression in the primary care setting: a randomized controlled trial. JAMA 2002;288:2836-45.

7. Katzelnick DJ, Williams MD. Large-scale dissemination of collaborative care and implications for psychiatry. Psychiatr Serv 2015;66:904-906.

8. Katon WJ, Von Korff M, Lin EH, et al. The pathways study: a randomized trial of collaborative care in patients with diabetes and depression. Arch Gen Psychiatry 2004;61:1042-9.

9. Rollman BL, Belnap BH, LeMenager MS, et al. Telephone-delivered collaborative care for treating post-cabg depression: a randomized controlled trial. JAMA 2009;302:2095-103.
10. Katon WJ, Lin EH, Von Korff M, et al. Collaborative care for patients with depression and chronic illnesses. N Engl J Med 2010;363:2611-20.

11. Schonlau M, Mangione-Smith R, Chan KS, et al. Evaluation of a quality improvement collaborative in asthma care: does it improve processes and outcomes of care? Ann Fam Med 2005;3:200-8.

12. Morgan MA, Coates MJ, Dunbar JA, Reddy P, Schlicht K, Fuller J. The trueblue model of collaborative care using practice nurses as case managers for depression alongside diabetes or heart disease: a randomised trial. BMJ Open 2013; Jan 24;3.

13. Woltmann E, Grogan-Kaylor A, Perron B, Georges H, Kilbourne AM, Bauer MS. Comparative effectiveness of collaborative chronic care models for mental health conditions across primary, specialty, and behavioral health care settings: Systematic review and meta-analysis. Am J Psychiatry 2012;169: $790-804$.

14. McAlister FA, Lawson FM, Teo KK, Armstrong PW. Randomised trials of secondary prevention programmes in coronary heart disease: systematic review. BMJ 2001;323:957-62.

15. Rossom RC, Solberg LI, Magnan S, et al. Impact of a national collaborative care initiative for patients with depression and diabetes and/or cardiovascular disease. Gen Hosp Psychiatry 2017;44:77-85.

16. Brownson RC, Colditz GA, Proctor EK, eds. Dissemination and implementation research in health: translating science to practice. Oxford, UK: Oxford University Press; 2018.

17. Flottorp SA, Oxman AD, Krause J, et al. A checklist for identifying determinants of practice: a systematic review and synthesis of frameworks and taxonomies of factors that prevent or enable improvements in healthcare professional practice. Implement Sci 2013;8:35.

18. Damschroder LJ, Aron DC, Keith RE, Kirsh SR, Alexander JA, Lowery JC. Fostering implementation of health services research findings into practice: a consolidated framework for advancing implementation science. Implement Sci 2009;4:50.

19. Chaudoir SR, Dugan AG, Barr CH. Measuring factors affecting implementation of health innovations: a systematic review of structural, organizational, provider, patient, and innovation level measures. Implement Sci 2013;8:22.

20. Peek CJ, The National Integration Academy Council. Lexicon for Behavioral Health and Primary Care Integration: Concepts and Definitions Developed by Expert Consensus. Vol AHRQ Publication No. AHRQ 13-IP0001-EF. Rockville, MD: Agency for Healthcare Research and Quality; April 2013.

21. Cohen DJ, Davis MM, Hall JD, Gilchrist EC, Miller BF. A Guidebook of Professional Practices for Behavioral Health and Primary Care Integration: Observations from Exemplary Sites. Vol AHRQ Publication No. 14-0070-1-EF. March ed. Rockville, 
MD: Agency for Healthcare Research and Quality; 2015. Available from: https://integrationacademy.ahrq.gov/sites/default/files/AHRQ_AcademyGuidebook.pdf. Accessed 06/20/2016.

22. Collins C, Hewson DL, Munger R, Wade T. Evolving models of behavioral health integration in primary care. New York., NY: Milbank Memorial Fund; May 2010.

23. Gallo JJ, Zubritsky C, Maxwell J, et al. Primary care clinicians evaluate integrated and referral models of behavioral health care for older adults: results from a multisite effectiveness trial (prism-e). Ann Fam Med 2004;2:305-9.

24. Strosahl K, Robinson P. The primary care behavioral health model: applications to prevention, acute care and chronic condition management. In: Kessler R, Stafford D, eds. Collaborative medicine case studies: evidence in practice. New York: Springer; 2008.

25. Blount A. Integrated primary care: organizing the evidence. Fam Syst Health 2003;21:121-33.

26. Petterson S, Miller BF, Payne-Murphy JC, Phillips RL. Mental health treatment in the primary care setting: patterns and pathways. Fam Syst Health 2014;32:157-66.

27. Doherty WJ, McDaniel SH, Baird MA. Five levels of primary care/behavioral healthcare collaboration. Behav Healthc Tomorrow 1996;5:25-7.

28. SAMHSA-HRSA, Center for Integrated Health Solutions. A standard framework for levels of integrated healthcare. April 2013; Available from: http://www.integration.samhsa.gov/integratedcare-models/A_Standard_Framework_for_Levels_ of_Integrated_Healthcare.pdf. Accessed 06/20/2016.

29. Miller BF, Talen MR, Patel KK. Advancing integrated behavioral health and primary care: the critical importance of behavioral health in health care policy. In: Talen MR, Valeras AB, eds. Integrated behavioral health in primary care: evaluating the evidence, identifying the essentials. New York, NY: Springer; 2013.

30. Solberg LI, Crain AL, Maciosek MV, et al. A stepped-wedge evaluation of an initiative to spread the collaborative care model for depression in primary care. Ann Fam Med 2015;13:412-20.

31. Whitebird RR, Solberg LI, Jaeckels NA, Pietruszewski PB, Hadzic S, Unutzer J, et al. Effective implementation of collaborative care for depression: what is needed? Am J Manag Care 2014;20:699-707.
32. Green LA, Cifuentes M. Advancing care together by integrating primary care and behavioral health. J Am Board Fam Med 2015;28 Suppl 1:S1-6.

33. Davis M, Balasubramanian BA, Waller E, Miller BF, Green LA, Cohen DJ. Integrating behavioral and physical health care in the real world: early lessons from advancing care together. J Am Board Fam Med 2013;26:588-602.

34. Coleman KJ, Magnan S, Neely C, et al. The compass initiative: description of a nationwide collaborative approach to the care of patients with depression and diabetes and/or cardiovascular disease. Gen Hosp Psychiatry 2017;44:69-76.

35. Coleman KJ, Hemmila T, Valenti MD, et al. Understanding the experience of care managers and relationship with patient outcomes: the COMPASS initiative. Gen Hosp Psychiatry 2017;44:86-90.

36. Kroenke K, Spitzer RL, Williams JB. The phq-9: validity of a brief depression severity measure. J Gen Intern Med 2001;16:606-13.

37. Glaser J, Laudel G. Life with and without coding: two methods for early-stage data analysis in qualitative research aiming at causal explanations. Forum: Qualitative Social Research, 2013;14(May; 2):Article 5.

38. Maietta RC. State of the art: integrating software with qualitative analysis. In: Curry L, Shield RR, Wetle TT, eds. In: Improving aging and public health research: qualitative and mixed methods. Vol 1st ed. Washington, D.C.: American Public Health Association; 2006.

39. Fleuren M, Wiefferink K, Paulussen T. Determinants of innovation within health care organizations: literature review and Delphi study. Int J Qual Health Care 2004;16:107-23.

40. Whitebird RR, Solberg LI, Crain LA, et al. Clinician burnout and satisfaction with resources in caring for complex patients. Gen Hosp Psychiatry 2017;44:91-95.

41. Chambers DA, Norton WE. The adaptome: advancing the science of intervention adaptation. Am J Prev Med 2016;51(4S2):S124-S131.

42. Cohen DJ, Balasubramanian BA, Davis M, et al. Understanding care integration from the ground up: five organizing constructs that shape integrated practices. J Am Board Fam Med 2015;28 Suppl 1:S7-20.

43. Dickinson WP. Strategies to support the integration of behavioral health and primary care: what have we learned thus far? J Am Board Fam Med 2015;28 Suppl 1:S102-106. 